\title{
Home Healthcare Clinicians' Perspectives on Electronic Health Records: A Qualitative Study
}

\author{
Mollie HOBENSACK ${ }^{\mathrm{a}, \mathrm{b}}$, Marietta OJO ${ }^{\mathrm{b}}$, Kathryn BOWLES ${ }^{\mathrm{b}, \mathrm{c}}$, \\ Margaret McDONALD ${ }^{\mathrm{c}}$, Jiyoun $\mathrm{SONG}^{\mathrm{a}}$ and Maxim TOPAZ ${ }^{\mathrm{a}, \mathrm{b}}$ \\ ${ }^{a}$ Columbia University School of Nursing, New York, New York, USA \\ ${ }^{\mathrm{b}}$ Visiting Nurse Services of New York, New York, USA \\ ${ }^{\mathrm{c}}$ University of Pennsylvania School of Nursing, Philadelphia, Pennsylvania, USA
}

\begin{abstract}
Clinicians' perspectives on the electronic health records (EHR) in home healthcare (HHC) are understudied. To explore this topic, qualitative interviews were conducted with $15 \mathrm{HHC}$ clinicians in the Northeastern USA. Thematic analysis was conducted to identify key themes emerging from the interviews. While some EHR benefits were recognized, overall satisfaction with the EHR was low. The results suggest EHR limitations are tied to poor usability, restrictions, and redundancy in documentation leading to increased documentation workload. Clinicians have recommendations to mitigate these limitations via additional EHR functions and better patient risk detection. Future stakeholders should consider the results of this study when developing and updating the EHR in HHC.
\end{abstract}

Keywords. Home healthcare, EHR, qualitative, satisfaction

\section{Introduction}

In 2017 , it was reported that $78 \%$ of home healthcare (HHC) agencies in the United States (USA) had adopted the electronic health record (EHR) [1]. The integration of an EHR was intended to improve patient safety, support patient care delivery, improve efficiency, and facilitate management of chronic conditions.

Additionally, EHRs are recognized as supporting information availability, interdisciplinary communication, and continuity of care [2]. However, the increase in EHR adoption came with an increased documentation burden [3]. Several studies have sought out clinicians' perspectives on the EHR and found that they reported ambivalence and low satisfaction regarding their EHR use [4-6].

In the US, home healthcare (HHC) agencies provide care to more than 5.4 million adults per year [9]. HHC services are provided for a limited amount of time (average length of stay is 22 days) [7]. The goal of HHC is to provide services related to skilled nursing, physical therapy, occupational therapy, speech therapy, and social work. Despite the growing importance of HHC services and technology use in all healthcare settings, there is limited knowledge from the clinicians' perspective on EHR use in HHC. Two early studies focused on 1) the tradeoffs nurses experience when using the EHR [8] and 2) the challenges that accompany EHR use in homecare [9]. To expand the findings of previous studies, this study aimed to explore HHC clinicians' perspectives and 
satisfaction with the EHR system used in their clinical practice with an ultimate goal of informing current and future EHR development efforts.

\section{Methods}

Study design and sample: This qualitative study is part of a parent study conducted at a large not-for-profit HHC agency located in the Northeastern USA. The agency employs approximately 2,300 clinicians and provides care to more than 75,000 patients annually in more than $900,000 \mathrm{HHC}$ visits. Clinicians were eligible to participate if they had more than 5 years of clinical experience in HHC. Study interviews were conducted from January 2021 to March 2021 with 15 clinicians. Clinicians were informed that their participation was voluntary and they could withdraw at any point during the interview. All study procedures were approved by the Institutional Review Board.

Data collection: The research team members with extensive informatics scholarship (research and publications) or HHC expertise (greater than 5 years) developed and revised the interview guide via several rounds of expert review and comments, until the final version was developed. The final interview guide included semi-structured and open-ended questions that covered aspects related to electronic documentation in HHC. During the one-time interview, clinicians were asked demographic questions (e.g., how many years of experience they had in HHC). Clinicians estimated the percentage of time they spent on documentation and rated their level of satisfaction with their current EHR on a scale from 0-10, with 10 indicating the highest level of satisfaction. Qualitative interviews were conducted by the principal investigator (MT), the research coordinator (MO), and the research assistant (MH) using the final interview guide. The interview audio files were transcribed into text for analysis.

Data analysis: Interview transcripts were imported into Dedoose, a web application used to analyze qualitative interviews [10]. Thematic analysis was conducted by two team members (MH, MO) referencing Kinger \& Varpio's Guide [11]. Both reviewers either participated in the original interview or had listened to the interview recording. This helped the two reviewers to familiarize themselves with the data. The two reviewers developed a codebook containing code names, code definitions, and "exemplars" linked to a unique identification (ID) to distinguish the quote owner. Then, the codes were extracted to identify emerging themes. Themes were discussed between the two reviewers and an additional team member until consensus was reached on the thematic category and definition.

\section{Results}

A total of 15 respondents participated in this study. Most participants had a Master's degree or higher $(60 \%)$, worked in the HHC setting for more than 6 years $(92 \%)$ and were nurses $(53 \%)$. While clinicians did recognize some of the benefits of EHR documentation, general satisfaction, on a scale where 10 represented high satisfaction, was relatively low with an average satisfaction of $5.3(\mathrm{SD}=2.1$, range $0-8)$. Clinicians self-reported their percentage of time spent on documentation during patient visits as $49.2 \%(\mathrm{SD}=12.5$, range $30 \%-70 \%)$.

Four themes related to clinician satisfaction with the EHR emerged: (1) usefulness, (2) limitations, (3) documentation workload, and (4) recommendations for EHR 
improvement. Within the theme usefulness, clinicians discussed the benefits of the EHRs in improving communication and highlighting key information. Multiple clinicians endorsed that all the information was available; however, finding the information they needed in a timely manner was difficult. Poor usability described a majority of clinicians' comments related to limitations of the EHR. This leads to documentation workload and a time burden especially depending on the clinicians' caseload and the acuity of the patients. To address these limitations, clinicians suggested adding functions and integrating patient risk identification alerts.

\section{Discussion}

This study used qualitative methods to explore HHC clinicians' perspectives and satisfaction of the EHR. Clinicians described several benefits of the EHR in HHC like its ability to "communicate with other clinicians." These results are supported by previous studies that identified team communication as a key usage of the EHR $[8,9]$. Specifically, in this study clinicians endorsed the use of documentation to highlight key information for other clinicians to know. Previous studies have discussed the association between documentation patterns and its prediction of adverse events [12,13]. In addition, clinicians recognized that a benefit of the EHR in HHC is that the "information is available." In comparison to previous modes where communication was primarily through phone calls or faxes, another study reported that nurses appreciate that the information is contained in a unified place (EHR) rather than patient information being stored in different locations [8].

While clinicians recognized some benefits of electronic documentation, a significant portion of their responses reflected overall low satisfaction with the EHR in HHC. Low clinician satisfaction with the EHR is not a new finding and has emerged across other studies [5,6]. In HHC one study found that clinicians were dissatisfied with the usability and functionality of the EHR [9]. The results of our study support these previous findings.

One of the most prevalent themes to arise in discussing limitations of the EHR in HHC was poor usability. Clinicians reported usability concerns related to searching through "many irrelevant notes" to find key information to inform patient care. Usability concerns with the EHR related to navigating and finding information was previously reported [9]. Additionally, clinicians discussed the restrictions and redundancy of the EHR as being a limitation to the EHR structure. Frustrations with restriction and redundancy [9] in documentation have been reported in previous studies in healthcare. Striking the balance between flexibility preferred by clinicians and structure by those using the data is a complex task [14]. This is often mitigated through documentation choices of structured or unstructured EHR data capture. However, this creates even more burden for clinicians to allocate time to sift through to identify important information they need at the point of care.

Another frequent theme was documentation workload. HHC clinicians spent about half of their working time documenting in EHR. This burden has been captured in other studies reporting that clinicians have decreased time for patient care due to documentation requirements $[3,9,15,16]$. Similarly, clinicians also spoke on how documentation workload increases based on complexity of the patient [17] and their assigned caseload [18]. Start of care documentation, completed at patient's admission to HHC, was specifically noted as being more time consuming compared to documenting 
other care activities. This can cause a delay in the initiation of patient care at the point of care [19] indicating the importance of streamlined, efficient EHRs.

Finally, this study offered participants the option to suggest recommendations for EHR improvement. A majority of the comments centered around additional functions being added to the EHR in HHC. These functions included the ability to search EHR data, explain referral rationale, and include the perspective of the patient. Similarly, several previous studies have sought to incorporate patient's values and perspectives in EHR data [20,21]. Another key suggestion focused on the addition of a function that might alert clinicians when patients experience concerning events that might lead to patient deterioration and negative outcomes. Although several studies have supported risk identification from EHR documentation in the HHC setting [22,23], little is known on the best approaches to identifying and sharing these concerning trends with HHC clinicians.

A limitation to this study was our small sample size and involving clinicians from only one USA-based HHC agency. While the study did include clinicians from multiple disciplines, future studies should engage larger samples with clinicians from different disciplines and additional HHC agencies.

\section{Conclusion}

This study explored HHC clinicians' perspectives and satisfaction with the EHR. Although some benefits of EHR were reported, clinicians' overall satisfaction was low and they spent about $50 \%$ of their time in the home on documentation. The results revealed limitations of the EHR are tied to poor usability, restriction, and redundancy in documentation leading to increased documentation workload. Clinicians have recommendations to mitigate these limitations via additional EHR functions and better patient risk detection. Future EHR development efforts in HHC can use these recommendations to improve the current and future systems.

\section{Acknowledgements}

This study was funded by the Agency for Healthcare Research and Quality (AHRQ) \#R01HS027742: "Building risk models for preventable hospitalizations and emergency department visits in homecare (Homecare- CONCERN)." The content is solely the responsibility of the authors and does not necessarily represent the official views of the Agency for Healthcare Research and Quality. This research was also supported by the National Institutes of Health through the funding of T32NR0769 (MH).

\section{References}

[1] Henry J, Plypchuck Y, Patel V. Electronic health record adoption and interoperability among U.S. skilled nursing facilities in 2017. ONC Data Brief. 41 (2018).

[2] Robles J. The effect of the electronic medical record on nurses' work. Creat. Nurs. 15 (2009) 31-35. doi:10.1891/1078-4535.15.1.31

[3] Gesner E, Gazarian P, Dykes P. The burden and burnout in documenting patient care: An integrative literature review. On: Stud. Health Technol. Inform., IOS Press, 2019: pp. 1194-1198. doi:10.3233/SHTI190415 
[4] Schenk E, Marks N, Hoffman K, Goss L. Four years later: Examining nurse perceptions of electronic documentation over time. J. Nurs. Adm. 51 (2021) 43-48. doi:10.1097/NNA.0000000000000965

[5] Top M, Gider Ö. Nurses' views on electronic medical records (EMR) in Turkey: An analysis according to use, quality and user satisfaction. J. Med. Syst. 36 (2012) 1979-1988. doi:10.1007/s10916-011-96576

[6] Topaz M, Ronquillo C, Peltonen LM et al. Nurse informaticians report low satisfaction and multi-level concerns with electronic health records: Results from an international survey. AMIA ... Annu. Symp. Proceedings. AMIA Symp. 2016 (2016) 2016-2025. https://pubmed.ncbi.nlm.nih.gov/28269961/ (accessed April 13, 2021)

[7] O'Connor M, Hanlon A, Naylor MD, Bowles KH. The impact of home health length of stay and number of skilled nursing visits on hospitalization among Medicare-reimbursed skilled home health beneficiaries. Res. Nurs. Heal. 38 (2015) 257-267. doi:10.1002/nur.21665

[8] De Vliegher K, Paquay L, Vernieuwe S, Van Gansbeke H. The experience of home nurses with an electronic nursing health record. Int. Nurs. Rev. 57 (2010) 508-513. doi:10.1111/j.14667657.2010.00827.x

[9] Sockolow PS, Bowles KH, Adelsberger MC, Chittams JL, Liao C. Challenges and facilitators to adoption of a point-of-care electronic health record in home care. Home Health Care Serv. Q. 33 (2014) 14-35. doi:10.1080/01621424.2013.870098

[10] Dedoose Version 8.0.35, web application for managing, analyzing, and presenting qualitative and mixed method research data (2018). Los Angeles, CA: SocioCultural Research Consultants, LLC.

[11] Kiger ME, Varpio L. Thematic analysis of qualitative data: AMEE Guide No. 131. Med. Teach. 42 (2020) 846-854. doi:10.1080/0142159X.2020.1755030

[12] Collins S. How extra nursing notes point to deterioration. Nurs. Times. 110 (2014) 19-20. https://pubmed.ncbi.nlm.nih.gov/24984372/ (accessed April 13, 2021)

[13] Topaz M, Woo K, Ryvicker M, Zolnoori M, Cato K. Home healthcare clinical notes predict patient hospitalization and emergency department visits. Nurs. Res. 69 (2020) 448-454. doi:10.1097/NNR.0000000000000470

[14] Rosenbloom ST, Denny JC, Xu H, Lorenzi N, Stead WW, Johnson KB. Data from clinical notes: A perspective on the tension between structure and flexible documentation. J. Am. Med. Informatics Assoc. 18 (2011) 181-186. doi:10.1136/jamia.2010.007237

[15] Guo U, Chen L, Mehta PH. Electronic health record innovations: Helping physicians - One less click at a time. Health Inf. Manag. 46 (2017) 140-144. doi:10.1177/1833358316689481

[16] Joukes E, Abu-Hanna A, Cornet R, De Keizer NF. Time spent on dedicated patient care and documentation tasks before and after the introduction of a structured and standardized electronic health record. Appl. Clin. Inform. 9 (2018) 46-53. doi:10.1055/s- 0037-1615747

[17] Prater L, Sanchez A, Modan G, Burgess J et al. Electronic health record documentation patterns of recorded primary care visits focused on complex communication: A qualitative study. Appl. Clin. Inform. 10 (2019) 247-253. doi:10.1055/s-0039-1683986

[18] Mutshatshi TE, Mothiba TM, Mamogobo PM, Mbombi MO. Record-keeping: Challenges experienced by nurses in selected public hospitals. Curationis. 41 (2018). doi:10.4102/curationis.v41i1.1931

[19] Yang Y, Bass EJ, Bowles KH, Sockolow PS. Impact of home care admission nurses' goals on electronic health record documentation strategies at the point of care. CIN - Comput. Informatics Nurs. 37 (2019) 39-46. doi:10.1097/CIN.0000000000000468

[20] Desai AV, Michael CL, Kuperman GJ et al. A novel patient values tab for the electronic health record: A user- centered design approach. J. Med. Internet Res. 23 (2021). doi:10.2196/21615

[21] Balhara KS, Millstein JH. Partners in narrative: Empowering patient-physician partnerships in the electronic health record. J. Patient Exp. 7 (2020) 833-835. doi:10.1177/2374373520962608

[22] Woo K, Adams V, Wilson P et al. Identifying urinary tract infection-related information in home care nursing notes. J. Am. Med. Dir. Assoc. (2021). doi:10.1016/j.jamda.2020.12.010

[23] Zolnoori M, McDonald MV, Barrón Y et al. Improving patient prioritization during hospital-homecare transition: Protocol for a mixed methods study of a clinical decision support tool implementation. JMIR Res. Protoc. 10 (2021). doi:10.2196/20184 